\title{
Targeting PKCI-PAK1 signaling pathways in EGFR and KRAS mutant adenocarcinoma and lung squamous cell carcinoma
}

Masaoki Ito ${ }^{1,2,3}$, Carles Codony-Servat ${ }^{1,4}$, Jordi Codony-Servat ${ }^{1}$, David Lligé2 ${ }^{2}$ Imane Chaib², Xiaoyan Sun ${ }^{5}$, Jing Miao ${ }^{5}$, Rongwei Sun ${ }^{5}$, Xueting $\mathrm{Cai}^{5}$, Alberto Verlicchi ${ }^{1,8}$, Morihito Okada ${ }^{3}$, Miguel Angel Molina-Vila?', Niki Karachaliou', Peng Cao ${ }^{5,6,7^{*}}$ and Rafael Rosell ${ }^{2,9,10^{*}}$

\begin{abstract}
Introduction: p21-activated kinase 1 (PAK1) stimulates growth and metastasis in non-small cell lung cancer (NSCLC). Protein kinase C iota (PKCI) is an enzyme highly expressed in NSCLC, regulating PAK1 signaling. In the present study we explored whether the PKCI-PAK1 signaling pathway approach can be an efficient target in different types of NSCLC cell and mouse models.

Methods: The effect of IPA-3 (PAK1 inhibitor) plus auranofin (PKCI inhibitor) combination was evaluated by cell viability assay, colony formation and western blotting assay, using three types of NSCLC cell lines: EGFR or KRAS mutant adenocarcinoma and squamous cell carcinoma with PAK1 amplification. In addition, for clinical availability, screening for new PAK1 inhibitors was carried out and the compound OTSSP167 was evaluated in combination with auranofin in cell and mice models.

Results: The combination of IPA-3 or OTSSP167 plus auranofin showed high synergism for inhibiting cell viability and colony formation in three cell lines. Mechanistic characterization revealed that this drug combination abrogated expression and activation of membrane receptors and downstream signaling proteins crucial in lung cancer: EGFR, MET, PAK1, PKCI, ERK1/2, AKT, YAP1 and mTOR. A nude mouse xenograft assay demonstrated that this drug combination strongly suppressed tumor volume compared with single drug treatment.

Conclusions: Combination of IPA-3 or OTSSP167 and auranofin was highly synergistic in EGFR or KRAS mutant adenocarcinoma and squamous cell carcinoma cell lines and decreased tumor volume in mice models. It is of interest to further test the targeting of PKCI-PAK1 signaling pathways in EGFR mutant, KRAS mutant and squamous NSCLC patients.
\end{abstract}

\section{Background}

Non-small cell lung cancer (NSCLC) is the leading cause of cancer related deaths and comprises several histological subtypes: lung adenocarcinoma (LUAD), squamous cell carcinoma (SCC) and large cell carcinoma. Despite the identification of targeted druggable driver mutations and rearrangements, most cases have poor survival [1, 2]. Recently, pembrolizumab plus

\footnotetext{
* Correspondence: njpcao@126.com; rrosell@iconcologia.net

${ }^{5}$ Hospital of Integrated Traditional Chinese and Western Medicine, Nanjing University of Chinese Medicine, Nanjing, China

${ }^{2}$ Laboratory of Cellular and Molecular Biology, Institute for Health Science

Research Germans Trias i Pujol (IGTP), Badalona, Spain

Full list of author information is available at the end of the article
}

chemotherapy have provided benefit in a fraction of patients, regardless of the level of PD-L1 expression [3-5]. However, the effect of immunotherapy in patients with EGFR mutations is rather limited [6]. A meta-analysis indicated that immuno-checkpoint inhibitors as second line treatment do not improve overall survival in comparison with docetaxel treatment in EGFR-mutant patients [7]. We focus our research on the identification of recurrent pathways occurring in subclasses of NSCLC, including LUADs driven by KRAS or EGFR mutations, and SCC. This stems from the fact that atypical protein kinase $\mathrm{C}$ binding to Par6 is associated with the epithelial cell transforming

(c) The Author(s). 2019 Open Access This article is distributed under the terms of the Creative Commons Attribution 4.0 International License (http://creativecommons.org/licenses/by/4.0/), which permits unrestricted use, distribution, and 
sequence 2 (Ect2), a guanine nucleotide exchange factor that activates Rac1 in downstream PAK1, MEK1/2ERK1/2 signaling, regulating tumor growth in NSCLC [8-10]. PKCı is reported to be amplified in $20.2-36.5 \%$ of NSCLC patients, especially in SCC patients $[8,11]$. PKCı mRNA is overexpressed in LUAD and SCC cell lines and tumor tissue, and is predictive of poor outcome [12]. The abundance of $\mathrm{PKCl}$ mRNA predicted sensitivity to an anti-rheumatoid agent, aurothiomalate, in a panel of lung cancer cell lines [8]. Auranofin, a gold complex used to treat rheumatoid arthritis was shown to inhibit the PI3K/AKT/mTOR signaling in NSCLC cell lines. The administration of auranofin to mice with xenograft tumors significantly suppresses tumor growth without inducing toxic effects [13]. It is of interest that auranofin enhances ibrutinib activity in EGFR mutant LUAD by inhibiting the expression or phosphorylation of multiple key nodes in AKT/mTOR and MEK/ERK pathways [14]. Furthermore, it has been demonstrated that $\mathrm{PKCl}$ plays an important function in KRAS LUADs $[15,16]$. PAK1 is a Ste20 (MAP $4 \mathrm{~K}$ ) member that is frequently overexpressed or amplified, and has a critical function in cell growth migration, invasion and apoptosis in NSCLC [17]. PAK1 confers cisplatin resistance in NSCLC patients [18]. PAK1 signaling has been shown to cause resistance to MAPK kinase inhibitors in BRAF mutant melanomas [19]. PAK1 mRNA expressing EGFR mutant tumors are resistant to EGFR tyrosine kinase inhibitors. The combination of gefitinib with an AKT inhibitor (perifosine) or PAK1 inhibitor (IPA-3) almost completely suppresses the tumor burden in nude mice harboring gefitinib resistant cells [20]. Together with others, we have demonstrated that the combination of AKT and EGFR inhibitors could be of benefit in patients with EGFR mutations [21] [22], however, even when blocking AKT, tumor regrowth could occur through the activation of other downstream regulators, via Src/FAK [22, 23]. We posit that different classes of LUADs, such as EGFR mutant LUADs, KRAS mutant LUADs and SCCs, could be sensitive to the inhibition of $\mathrm{PKCl}$ with auranofin plus a PAK inhibitor. Several attempts have been made to specifically target PAK1 in cancer, however, its catalytic pocket is large and highly flexible, in addition to its highly mobile $\mathrm{N}$-terminal lobe, which presented a challenge in preventing specific PAK inhibitors $[24,25]$. For this reason, we used IPA-3 (2,2'-dihydroxy-1,1' -dinaphthyldisulfide) as a PAK inhibitor [26] with $\mathrm{PKCl}$ inhibitor.

This study aimed to estimate the therapeutic effect of PKCI-PAK1 signaling pathways in different types of NSCLC.

\section{Materials and methods}

Detail of standard methodologies for cell culture, reagents, cell viability assay, colony formation assay, and western blotting analysis are described in Supplementary materials and methods.

\section{In vitro screening for PAK1 inhibitor}

The measurement of PAK1 kinase was performed on a Caliper LabChip EZ Reader II equipped with a 12-sipper chip in Profiler Pro separation buffer supplemented with CR-8 and analyzed using EZ Reader software (Caliper Life Sciences; Hopkinton, MA, USA). The test compounds were incubated with PAK1 kinase and fluorescence-labeled substrate Peptide 14 (Sequence RRRLSFAEPG) in the kinase assay buffer at $30^{\circ} \mathrm{C}$ for $10 \mathrm{~min}$, followed by adding $9.5 \mu \mathrm{M}$ ATP to initiate the reaction. After incubation for 1 h at $30^{\circ} \mathrm{C}$, the phosphorylated and unphosphorylated substrates were separated and detected by EZ Reader II device. IPA-3 was used as a positive control. The separation of peptide 14 was performed under the following optimized conditions: upstream voltage $=-500 \mathrm{~V}$, downstream voltage $=-2400 \mathrm{~V}$ and pressure $=-1.4$ psi using a marker dye consisting of unphosphorylated peptide 14. After PAK1 inhibitors were screened from the Target Mol-InhibitoryLibrary, the anti-tumor effects of candidates were estimated by MTT assay using A549 cell lines.

\section{Nude mouse xenograft}

Four to five-week-old female nude mice were kept in individually ventilated cages ( 5 per cage) with access to food and water, at $20^{\circ} \mathrm{C}$ and $50 \pm 20 \%$ relative humidity under a 12:12 h light-dark cycles and pathogen free conditions. Three cell line xenograft tumors were established by subcutaneously injecting $4 \times 10^{6}$ cells suspended in phosphate-buffered saline mixed 1:1 with Corning Matrigel (356,237, Corning, NY, USA) via right flank. Tumor size was measured in two orthogonal directions using calipers every 2 days and weights were determined every 2 days. After tumors became palpable $\left(\sim 100-300 \mathrm{~mm}^{3}\right)$, mice were randomized into a vehicle group and treated groups with IPA-3 alone, auranofin alone, OTSSP167 alone, IPA-3 plus auranofin, or OTSSP167 plus auranofin. Each reagent was suspended in $1 \%$ [weight/volume (w/v)] Kolliphor HS15 and administered once daily by intraperitoneal administration (IPA-3 and auranofin) or oral gavage (OTSSP167) of 10 $\mathrm{mg} / \mathrm{kg}$. Mice in the untreated group were given the same volumes of $1 \%$ Kolliphor HS15. The tumor volume $\left(\mathrm{mm}^{3}\right)$ was estimated using the equation length $\times$ $(\text { width })^{2} \times 0.5$.

\section{Statistical analysis}

In MTT and colony forming assays, the strength of interaction between reagents was determined by calculating the combination index (CI) according to method of isobologram-combination index (Chou-Talalay method [27]). The $\mathrm{CI}<0.6,0.6<\mathrm{CI}<0.8,0.8<\mathrm{CI}<0.9,0.9<\mathrm{CI}<$ 
1.1, 1.1 and $>1.1$ indicates strong synergism, moderate synergism, slight synergism, additive interaction, and antagonism, respectively. In mice xenograft experience, volume and weight of tumor and mice body weight were compared as continuous variables by using MannWhitney $\mathrm{U}$ tests. Two-sided statistics was employed and a $p$ value of less than 0.05 was regarded as significant.

\section{Results}

The combination of a PAK1 inhibitor plus antitumoral compounds showed cell viability and colony formation inhibition synergy

To investigate the potential antitumoral properties and possible synergistic effect of a PAK inhibitor (IPA-3) combination with several compounds used in cancer treatment, such as EGFR inhibitors (osimertinib and afatinib), PKCı inhibitor (auranofin), MEK inhibitor (trametinib), and a Flk3/Syk, and potential multiple PKC inhibitor (midostaurin), several lung cancer cell lines possessing different molecular characteristics were tested, such as HCC827 (EGFR mutated), H23 (KRAS mutated) and H520 (PAK1 overexpression). We evaluated cell viability and colony formation inhibition. Results indicated that the IPA-3 plus osimertinib combination showed synergism in $\mathrm{H} 23$ cell lines in cell viability assays with a $\mathrm{CI}$ of 0.73 . IPA-3 plus trametinib was also synergistic in the $\mathrm{H} 23$ cell line (CI was 0.74 )
(Fig. 1a). To highlight, IPA-3 plus PKCı inhibitor, auranofin, showed the highest synergism in each of the 3 cell lines (CI ranged 0.34 to 0.39 ) regardless of difference in histology or genetic profile (Fig. 1). IPA-3 plus midostaurin was slightly synergistic or addictive in 3 cell lines. The CIs of each combination treatment were shown in Table 1. The IC50s of each inhibitor in 3 cell lines are shown in Additional file 1: Table S1.

Furthermore, dose-dependent colony formation assays indicated that the combination of IPA-3 plus auranofin significantly inhibited the forming of colonies, compared to single agent treatment and showed high synergism in 3 cell lines (lowest CI ranging from 0.24-0.46) (Fig. 2 and Additional file 1: Figure S1). The values of quantification of crystal violet concentration are shown in Fig. $2 \mathrm{~b}$ and Additional file 1: Figure S1B/S1E. The combination index value was calculated to assess the nature of drug-drug interactions and it decreased in a dosedependent manner (Fig. 2c and Additional file 1: Figure $\mathrm{S} 1 \mathrm{C} / \mathrm{S} 1 \mathrm{~F})$.

\section{The combination of a PAK1 inhibitor plus antitumoral compounds downregulated the expression of proteins} related to deregulated signaling pathways in lung cancer To study the mechanisms involved in the synergism between IPA-3 and auranofin, we analyzed the effects of this combination in the expression and phosphorylation

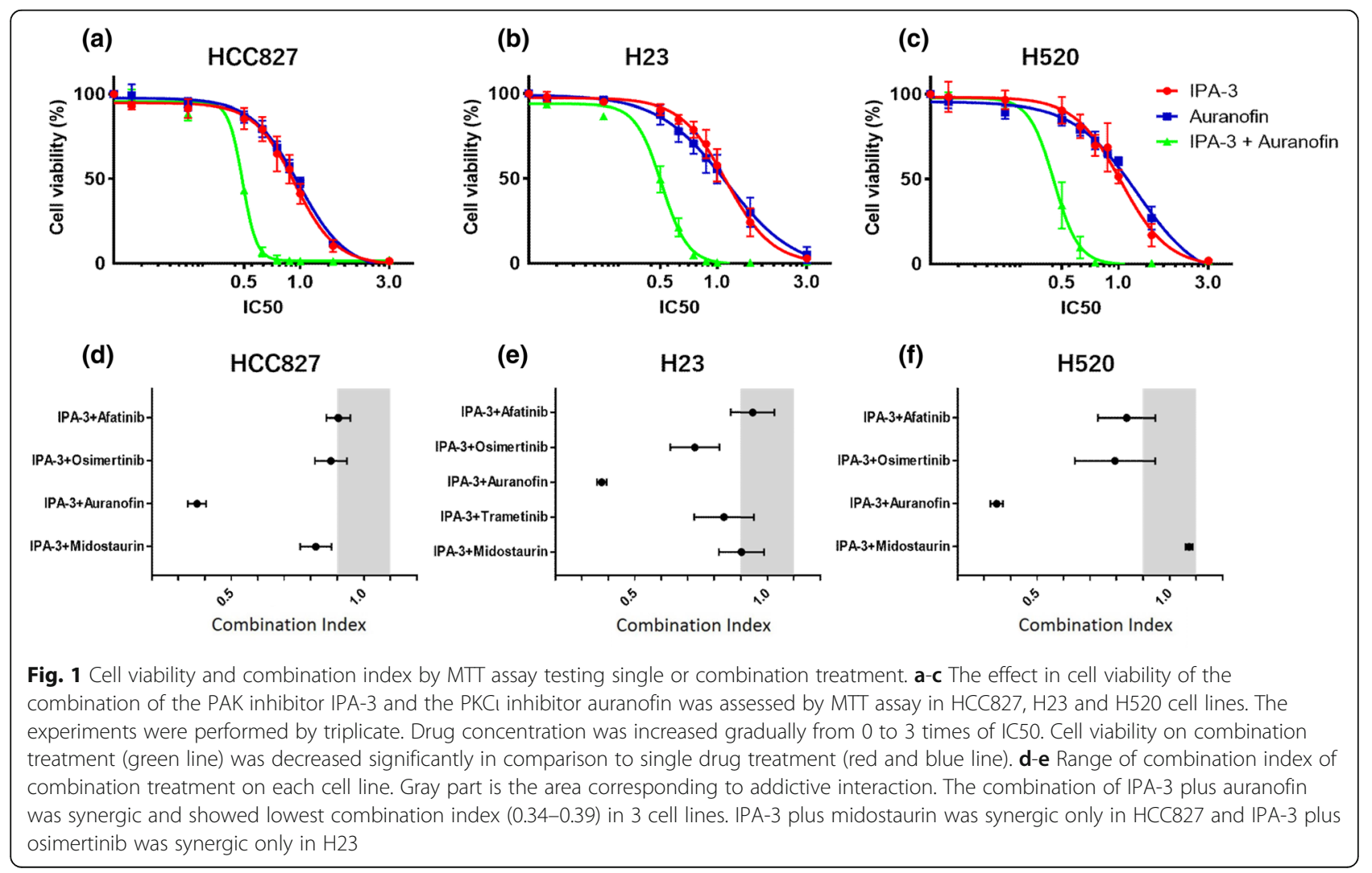


Table 1 Combination index of regents in 3 cell lines

\begin{tabular}{lcccccc}
\hline & IPA-3 & IPA-3 & IPA-3 & IPA-3 & IPA-3 & Osi \\
& + & + & + & + & + & + \\
& Afa & Osi & Aura & Mido & Tra & Aura \\
\hline HCC827 & 0.951 & 0.876 & 0.371 & 0.819 & & 1.123 \\
H23 & 0.944 & 0.727 & 0.376 & 0.902 & 0.836 & 0.929 \\
H520 & 0.838 & 0.794 & 0.347 & 1.073 & & 0.766 \\
\hline
\end{tabular}

Abbreviations: Afa afatinib, Aura auranofin, Mido midostaurin, Osi osimertinib, Tra trametinib

of proteins that belong to several signaling pathways related to lung cancer. Cell lysates were harvested after being treated with the indicated drug concentrations for $6 \mathrm{~h}$ and subjected to western blotting analysis. Results showed that the combination of IPA-3 plus auranofin abrogated the expression of total/phosphorylation of PAK1, PKCl, ERK 1/2, mTOR, AKT, and YAP1 in the 3 lung cancer cell lines studied HCC827 (EGFR-mutant LUAD), H23 (KRAS-mutant LUAD) and H520 (PAK1 overexpression). Also, downregulation of protein expression of EGFR, MEK, AXL, Src and STAT3 was observed. (Fig. 2 and Additional file 1: Figure S2). In summary, there is a clear downregulation of proteins, and, in most of them, a clear decrease in phosphorylation was detected when combining IPA-3 plus auranofin, indicating that this drug combination may target multiple signaling pathways. In addition, we investigated the molecular effects of auranofin combined with osimertinib, an EGFR inhibitor used in EGFR-mutated lung cancer patients. The drug combination was tested in the EGFR-mutated cell line, HCC827, at the indicated dose for a period of $6 \mathrm{~h}$. Protein lysates were analyzed by western blotting technique which showed a clear protein expression decrease in AKT, MET, AXL and CDCP1 (Fig. 2). We carried out similar experiments with the combination of auranofin plus trametinib, a MEK inhibitor, in the KRAS mutated cell line, $\mathrm{H} 23$, and observed a clear downregulation of PAK1, PKCl, EGFR, ERK 1/2, AKT, MET, AXL, STAT3, and MEK 1/2. Inhibition of phosphorylated PAK1 (Ser144), PKCl, ERK 1/2, STAT3 and MEK 1/2 was also observed.

\section{Screening of alternative PAK1 inhibitor and validation of combination therapeutic effect with auranofin}

To identify pharmacological inhibitors of PAK1 kinase activity, we screened the Target-Mol inhibitor library based on an optimized LabChip EZ Reader kinase assay system. Titration and validation of the kinase assay are shown in Additional file 1: Figure S3. Out of 320 compounds tested, 13 candidates showed PAK1 inhibitory effect. The details and inhibition ratio of each compound are shown in Additional file 1: Table S2 and Additional file 1: Figure S4. Among them, the PAK1 inhibitory effects of some compounds, such as bosutinib (1-A5), WH-4-023 (3-
F9), and MHY1485 (4-H9), were comparable to IPA-3, and their inhibitory effect on cell growth was estimated using the MTT assay. Cell viability tested in A549 cell lines showed that 5 compounds (bosutinib, thonzonium bromide, AT13148, OTSSP167, and reversine) significantly suppressed cell growth at the concentrations of 10 and $20 \mu \mathrm{M}$ (Fig. 3).

Furthermore, among 5 potential PAK1 inhibitors, OTSSP167 plus auranofin showed synergism in 3 cell lines. CIs in MTT assay were $0.78-0.79$. The protein expressions of phosphorylated PAK1 were suppressed by $\times 0.5, \times 1.0$, and $\times 3.0$ times higher dose of IC50 (Fig. 4). Reversine indicated synergism with auranofin only in H520 cell lines (CI: 0.76). The IC50 and CI of OTSSP167 or reversine plus auranofin are shown in Table 2. Colony formation assay showed synergistic effect of OTSSP167 plus auranofin in 3 cell lines with lowest $\mathrm{CI}$ ranging from 0.50-0.59 (Fig. 5a-c and Additional file 1: Figure S5). Western blotting experiments showed that OTSSP167 inhibited more PAK1 phosphorylated residues compared to IPA-3. The combination of OTSSP167 plus auranofin abrogated phosphorylated PAK1 (Thr432 and Ser144 in 3 cell lines) (Fig. 5d). In addition, the combination of these two drugs downregulated the $\mathrm{PKC}$ expression and inhibited its phosphorylation.

The combination of PAK1 and PKCI inhibitors suppressed tumorigenesis in a nude mouse Xenograft model

We performed nude mice xenograft experiments to explore the pharmacological combined effect of PAK1 and PKCl inhibitors. HCC827, H23, or H520 cell lines were inoculated subcutaneously into mice and when tumor size reached an average volume of $100-300 \mathrm{~mm}^{3}$, single reagent (IPA-3, OTSSP167, auranofin) or combination of IPA-3 or OTSSP167 plus auranofin or vehicle were inoculated via intraperitoneal administration (auranofin and IPA-3) or oral gavage (OTSSP167) every 2 days for 2 or 4 weeks. Combination therapy showed stronger anti-tumor effect compared to mono therapy in 3 mice model cell lines. IPA3 plus auranofin inhibited tumor growth, both in volume and weight, compared to IPA-3 alone or auranofin alone. The combination of OTSSP167 plus auranofin showed significant inhibition of tumor growth in the 3 mice model cell lines compared to single OTSSP167 or auranofin treatment (Fig. 6 and Additional file 1: Figure S6). Compared to vehicle group, mice body weight was significantly reduced in OTSSP167 plus auranofin and single OTSSP167 treatment in the 3 mice model cell lines. The combination of IPA-3 plus auranofin induced significant body weight loss in HCC827 and H520 cell lines mice models.

\section{Discussion}

Inactive PAK1 is reported to be a homodimeric protein. Binding of $\mathrm{Cdc} 42 / \mathrm{Rac} 1$ to $\mathrm{Cdc} 42 / \mathrm{Rac} 1$-binding 


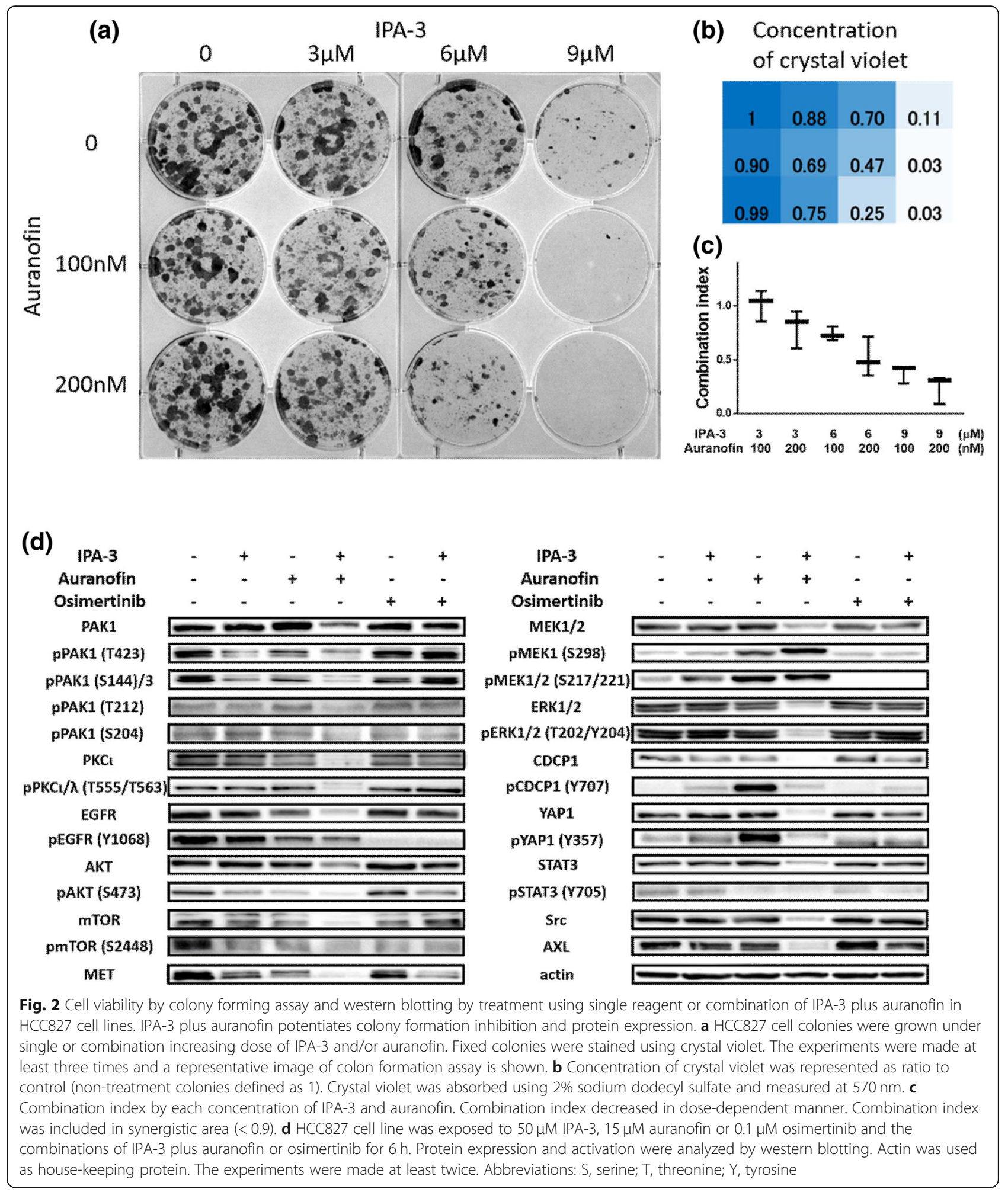

domain (CRIB) relieves inhibition by disrupting the PAK1 homodimer [28]. PAK1 is activated by several mechanisms that include $\mathrm{PKCl}$ commonly upregulated in NSCLC $[12,29]$, such as TNF $\alpha$, CD3/CD28 (T cell receptor engagement). EGFR signaling actively suppresses TNF mRNA levels by inducing expression of miR-21. EGFR TKIs result in loss of miR-21 and increase TNF mRNA stability, leading to EGFR TKI resistance in EGFR mutant NSCLC [30]. Therefore, it is tempting to speculate that an EGFR TKI, PAK 
<smiles>COc1cc(Nc2c(C#N)cnc3cc(OCCCN4CCN(C)CC4)c(OC)cc23)c(Cl)cc1Cl</smiles>

(d)<smiles>CC(=O)C1=CNC2=C(C(=NC3CCC(CN(C)C)CC3)N2)C1=C1C=C(Cl)C(=O)C(Cl)=C1</smiles>
3-A7
OTSSP167 (b)

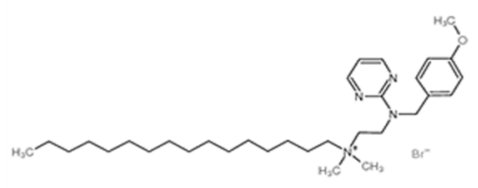

$1-\mathrm{C} 10$ THONZONIUM BROMIDE

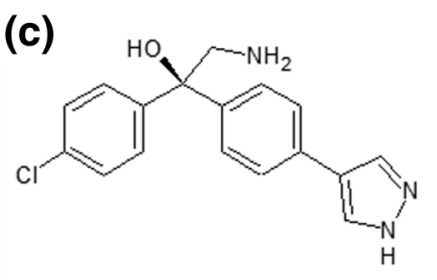

2-C11

AT13148
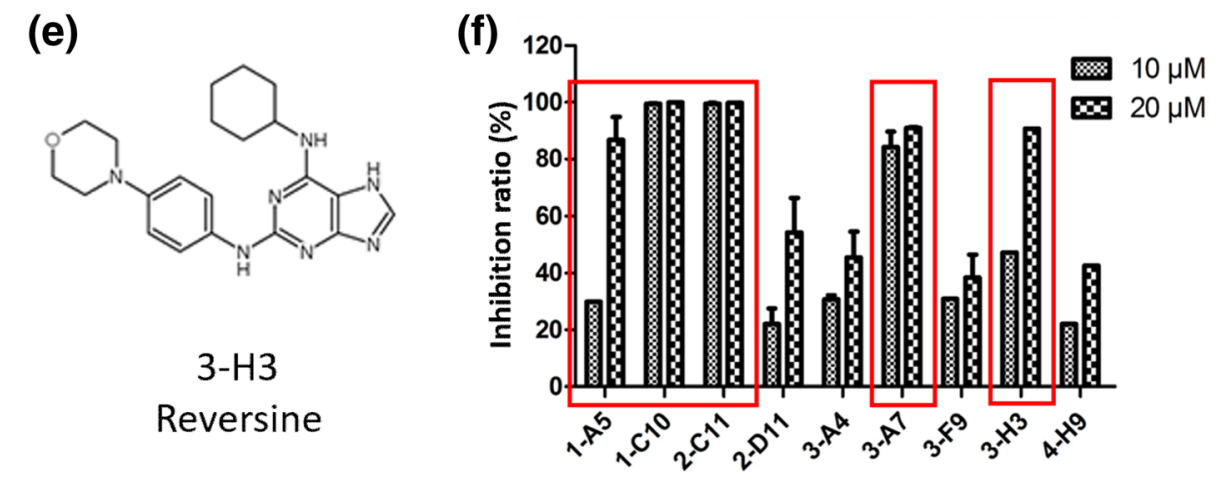

Fig. 3 Structures and cell growth inhibitions of the selected PAK1 inhibitors tested in A549 cell lines based on the preliminary screening (Additional file 1: Figure S4). The effect in cell viability of the potential PAK inhibitors. A549 cells were incubated with the 9 compounds at the concentration of 10 or $20 \mu \mathrm{M}$ for $72 \mathrm{~h}$ and the cell viability was determined using MTT assay. Five compounds (1-A5, 1-C10, 2-C11, 3-A7, and 3H3) significantly suppressed the growth of A549 cell lines at the range concentration of 10 and $20 \mu \mathrm{M}$. Statistical analysis was performed by comparing with the value of control. The experiments were made by triplicate. a-e The structures of 5 compounds which suppressed the cell viability. $\mathbf{f}$ Cell inhibition ratio on tested 9 compounds. Five compounds surrounded red square showed high PAK1 inhibition

inhibitor combination could serve as novel combinatory therapy for EGFR mutant NSCLC. The development of PAK1 inhibitors is difficult due to their large and highly flexible catalytic pocket and highly mobile N-terminal lobe [29, 31, 32]. PAK inhibitor combinations with targeted drugs have been tested in NSCLC cell lines, including apoptosis protein inhibitors (IAP, EGFR, MEK1/2 and Src inhibitors with PAK1 knockdown) [33]. Strong combinatorial activity was confirmed for dual inhibition of PAK1 and IAP in EBC-1 cells [33]. Activity was demonstrated with an inhibitor targeting PAK1 activation-3' (IPA-3) [33]. However, the cardiac toxicity noted with IPA-3 prevents its clinical use [34]. PAK1 serves as a mediator of intracellular calcium ion homeostasis in the heart. PAK1 inhibition induces exaggeration of calcium ion and arrhythmia $[35,36]$. Although it is unknown whether the toxicity is reversible or not, the risk can be reduced by lower doses of IPA-3 [34]. An auranofin phase I study for rheumatoid arthritis patients [37] and a randomized multicenter study for asthma patients [38] have reported no cardiac toxicity. Our study suggested that the dose of IPA-3 can be decreased in combination with auranofin. Nevertheless, cardiac toxicity is a major concern and attention should be paid to safety use in PKCl-PAK1 signaling strategy. We tested the combination of IPA-3 plus other inhibitors in cell models. IPA-3 plus auranofin showed high synergism in cell viability and colony formation assays in three NSCLC cell lines used. The inhibitory effect was superior to other combinations, such as EGFR tyrosine kinase inhibitors (either osimertinib or afatinib) in the HCC827, or MEK inhibitor (trametinib) in the $\mathrm{H} 23$ cell line. Also, in the SCC cell line, the combination index was significant for IPA-3 plus auranofin. Auranofin has already been clinically available for rheumatoid arthritis and a clinical trial for NSCLC and small cell lung cancer is ongoing (NCT01737502). Another PKC inhibitor, gold compound aurothiomalate, is also clinically available for rheumatoid arthritis and a phase I study has been successfully performed for advanced NSCLC [39]. However, IPA-3 has never been clinically used so we explored a PAK1 inhibitor with potential clinical availability.

OTSSP167 is a maternal embryonic leucine-zipper kinase (MELK) inhibitor with anti-cancer effect reported in several tumors, as well as in chronic lymphocytic leukemia [40]. The anti-tumor effects of OTSSP167 have been investigated in breast cancer (NCT02926690) and leukemia (NCT0279 5520) clinical trials [40-42]. OTSSP167 [41] inhibited 


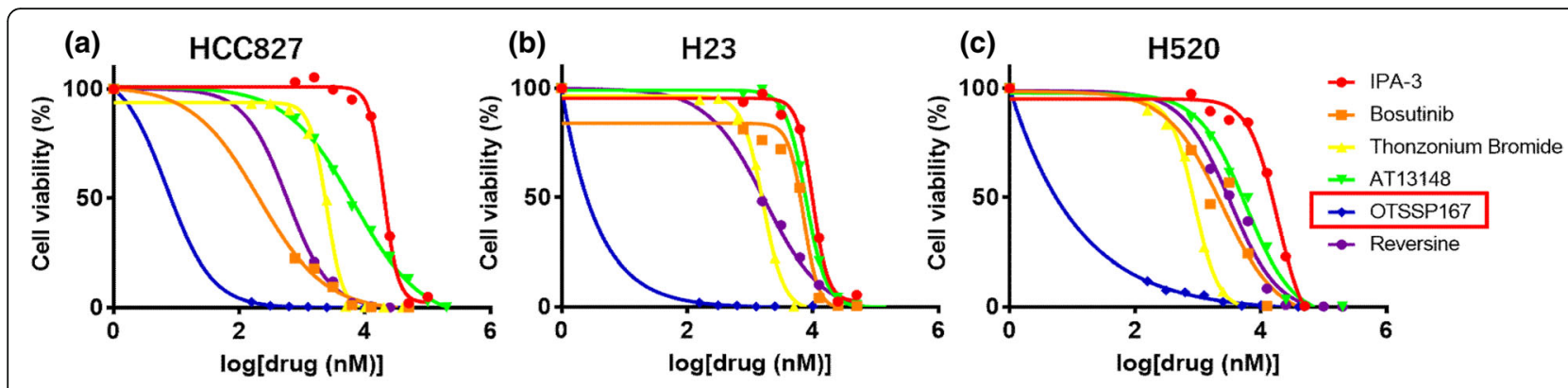

(d)

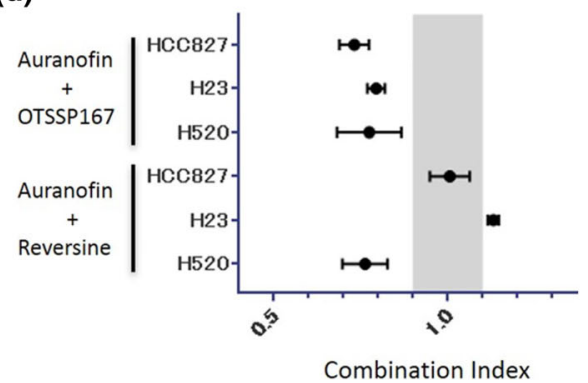

(e)

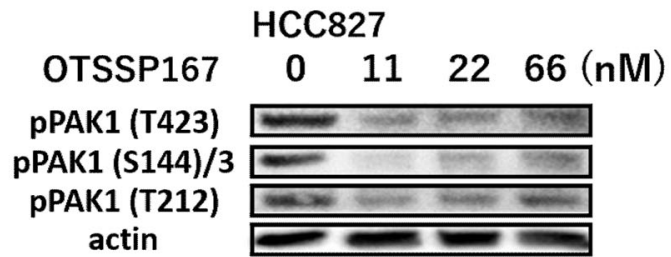

Fig. 4 Cell viability assay using potential PAK inhibitors. The potential PAK inhibitor OTSSP167 synergizes with auranofin in cell viability assays. a-c The results of MTT assay using alternative PAK1 inhibitors candidates in 3 cell lines: HCC827 (a), H23 (b), and H520 (c). OTSSP167 showed the lowest IC50 in 3 cell lines (blue line). (d) Combination index of OTSSP167 plus auranofin indicated synergism in 3 cell lines. The combination of reversine plus auranofin also indicated synergism in H520 cell lines but not in HCC827 and H23 cell lines. (e) OTSSP167 inhibited 3 phosphorylated PAK1 residues at the concentration of 0.5, 1.0, and 3.0 times higher of IC50

PAK1 with lower IC50 than other potential PAK1 inhibitors, such as bosutinib [43], thonzonium bromide [44], AT13148 [45] and reversine [46] (Fig. 4) and showed high synergism with auranofin in all three cell lines.

The phosphorylated residue Ser144 of PAK1 was inhibited in three cell lines. The phosphosites, Ser21 or Thr423, also contribute towards PAK1 activation, although, Ser144 is the most critical for PAK kinase activity [47]. Phosphorylated Ser144 on PAK1 was abrogated by IPA-3 plus auranofin, as well as by OTSSP167 plus auranofin.

Our model showed that some PAK1 phosphosites were inhibited by, not only IPA-3, but also auranofin alone. IPA-3 did not inhibit PKCı expression. This suggests that auranofin inhibits PAK1 from the upper stream of PKCL-PAK1 signaling and stronger inhibition of PKCI-PAK1 signaling resulted in stronger

Table 2 IC50 (nM) or combination index (Cl) of each single treatment or combination treatment in 3 cell lines

\begin{tabular}{ccccc}
\hline & Reagent & HCC827 & H23 & H520 \\
\hline \multirow{2}{*}{ IC50 } & OTSSP167 & 22 & 18 & 13 \\
& Reversine & 350 & 1300 & 5300 \\
Cl & OTSSP167 + Aura & 0.777 & 0.795 & 0.783 \\
& Reversine + Aura & 1.063 & 1.130 & 0.795 \\
\hline
\end{tabular}

Abbreviations: Aura auranofin synergism. Although osimertinib monotherapy in HCC827 cell lines or trametinib monotherapy in H23 cell lines also inhibited some phosphorylated PAK1, the combination of auranofin plus these reagents was not synergistic and showed less abrogation of RTK and non-RTK. In addition, midostaurin, a FLT3 inhibitor, and a potential inhibitor of all PKC isoforms [48] was not synergistic with auranofin (Fig. 1). In addition, although OTSSP167 served as a MELK inhibitor, inhibition of MELK expression was similar between OTSSP167 alone, and OTSSP167 plus auranofin (Fig. 5d), suggesting that synergism in OTSSP167 plus auranofin was not induced by MELK inhibition. Thus, PKCı-PAK1 signaling is an important pathway in tumor genesis in EGFR mutant, KRAS mutant and SCC cell lines. Additionally, due to the inhibition of most proteins analyzed by the auranofin plus IPA-3 or OTSSP167 combination, we hypothesize that this compound combination could affect the proteasome activity as previously reported [49].

In the mice xenograft model, a significant decrease in tumor volume was confirmed by the OTSSP167 plus auranofin combination. Although IPA-3 plus auranofin showed anti-tumor effect, it did not reach a significant difference. OTSSP167 suggested stronger PAK1 inhibition compared to IPA-3 in western blotting; phosphorylated PAK1 at Thr423/Ser144/Thr204 in HCC827 cell 


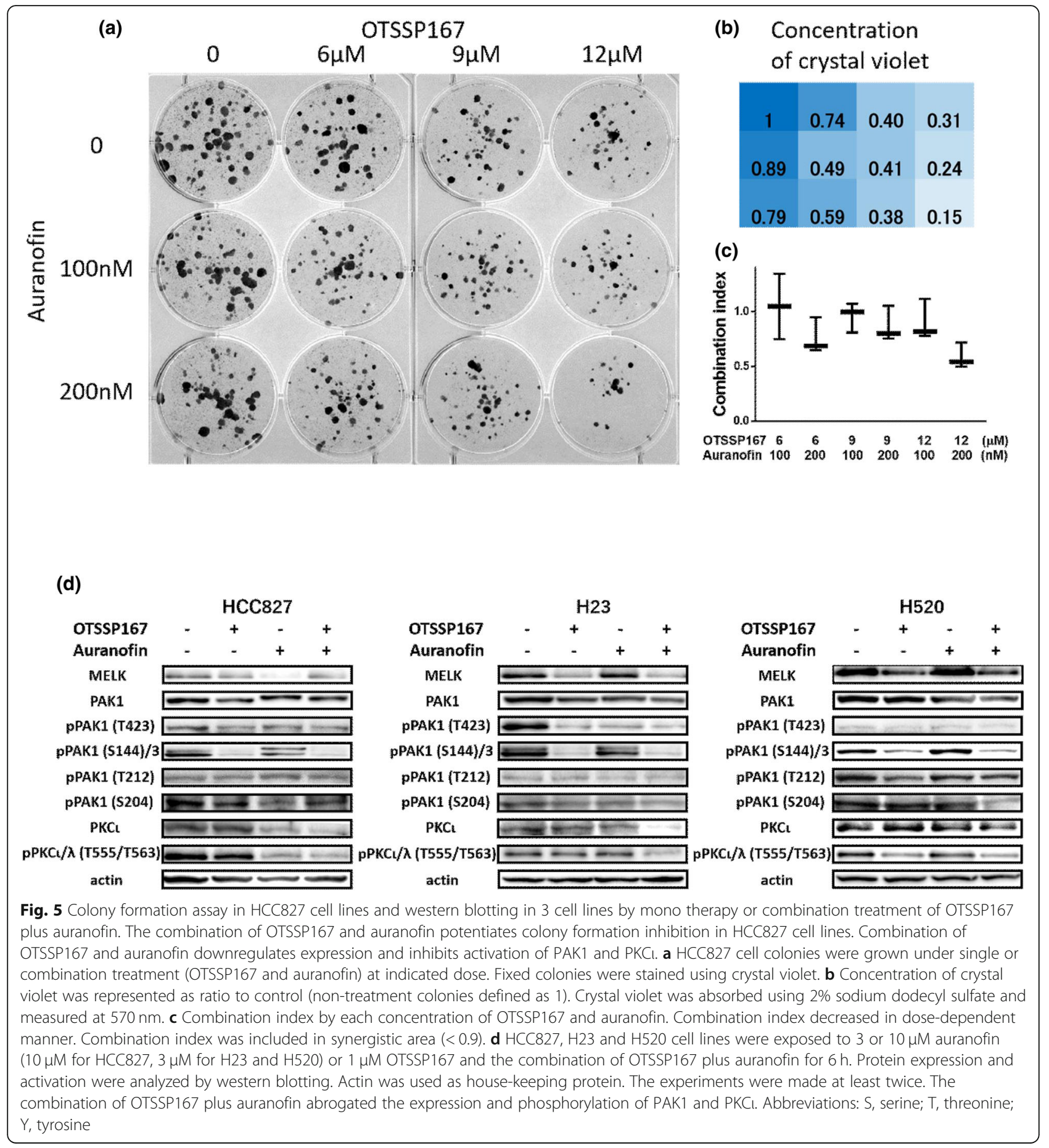

lines and Thr423 in $\mathrm{H} 23$ cell lines were inhibited by OTSSP167 alone, but not by IPA-3 alone. These differences between IPA-3 and OTSSP167 might explain the difference in results of the mice xenograft model. Although mice body weight was significantly decreased in OTSSP167 alone, or OTSSP167 plus auranofin in all 3 cell lines, there was no significant difference between
OTSSP167 alone, and OTSSP167 plus auranofin. Chung et al. showed OTSSP167 was tolerable in the mice model without adverse body weight loss [41]. Adverse events of OTSSP167 should be further examined.

Our research has centered on targeting PKCI-PAK1 signaling and was effective with auranofin plus OTSSP167 in 3 lung cancer models in vitro and in vivo. Although 


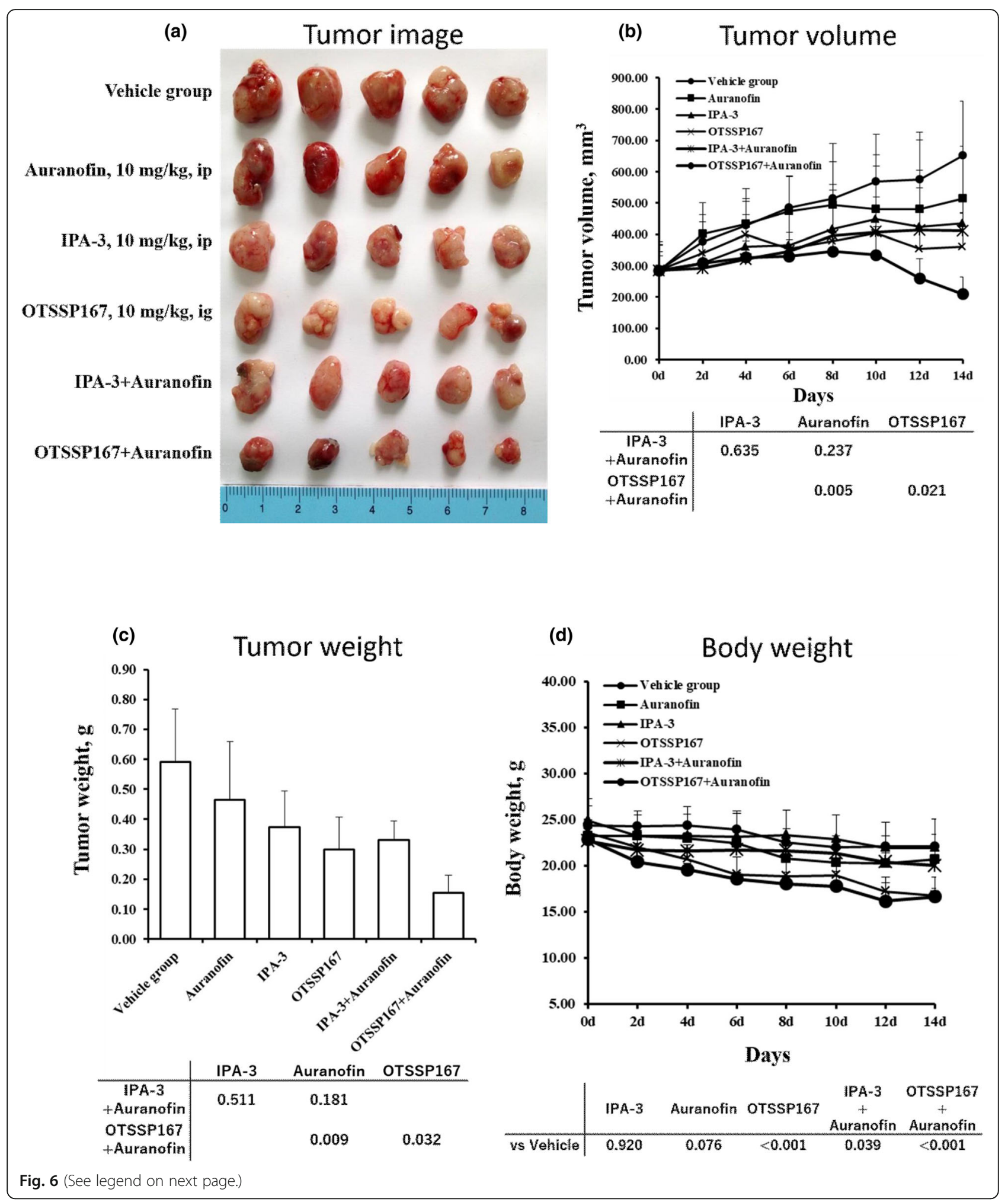


(See figure on previous page.)

Fig. 6 Anti-tumor effects of single reagent or combination treatment using IPA-3, auranofin, and OTSSP167 in nude xenograft model in HCC827. a HCC827 cell lines were used for the xenograft model. Mice were treated once daily with the PAK1 inhibitor (IPA-3 or OTSSP167) single agent or combined with auranofin at given concentrations. Representative images of tumors measured at the end of the study compared with the control group are shown. $\mathbf{b}$ Line graphs of tumor volumes after treatment by each therapeutic course (vehicle, single reagent, or combination). Tumor volumes were recorded every 2 days until 14th or 26th day. Errors bars mean +- SD of 5 animals per group. $P$ values by comparing tumor volumes are described in the table. $\mathbf{c}$ Histographs of tumor weight treated by each therapeutic course (vehicle, single reagent, or combination) at the time of sacrifice. $P$ values by comparing tumor weight are described in the table. $\mathbf{d}$ Line graphs of mice weight on every 2 days of each treatment course (vehicle, single reagent, or combination). $P$ values by comparing mice body weight are described in the table.

OTSSP167 inhibited phosphorylated PAK1, we cannot rule out that other activators of PAK1 can intervene in lung cancer, like MLK3 and TNF $\alpha$.

Auranofin has been investigated in a clinical trial for NSCLC and small cell lung cancer (NCT01737502). A Phase I study of OTSSP167 for solid tumors (NCT01910545) and a feasibility evaluation for healthy volunteers (NCT027685 19) have been completed. Other clinical trials of OTSSP167 are ongoing in breast cancer (NCT02926690) and leukemia (NCT02795520). Targeting PKCi-PAK1 signaling pathways is of interest to be further tested clinically in EGFR mutant, $K R A S$ mutant, and squamous NSCLC patients.

\section{Conclusions}

The Combination of PKCL-PAK1 inhibitors was highly synergistic in EGFR and KRAS mutant adenocarcinoma and squamous cell carcinoma of the lung, in both in vitro and in vivo mice models. It is warranted to further test the therapeutic strategy of targeting PKCl-PAK1 signaling pathways in EGFR mutant, KRAS mutant and squamous NSCLC patients.

\section{Additional file}

Additional file 1: Table S1. Profiles of cell lines and IC50 (nM) of each reagent. Table S2. The profiles of tested potential PAK1 inhibitors. Figure S1. IPA-3 and auranofin potentiates colony formation inhibition in $\mathrm{H} 23$ and $\mathrm{H} 520$ cell lines. Figure $\mathbf{S 2}$. Western blotting by treatment using single reagent or combination of IPA-3 plus auranofin in $\mathrm{H} 23$ and $\mathrm{H} 520$ cell lines. Figure S3. Optimization and validation of the kinase assay for PAK1 inhibitor screening. Figure S4. Summary of cell growth inhibition using potential PAK1 inhibitors. Figure S5. OTSSP167 plus auranofin potentiates colony formation inhibition in $\mathrm{H} 23$ and $\mathrm{H} 520$ cell lines. Figure S6. Anti-tumor effects of single reagent or combination treatment using IPA-3, auranofin, and OTSSP167 in nude xenograft model in $\mathrm{H} 23$ and $\mathrm{H} 520$ cell lines.

\section{Acknowledgements}

Not applicable.

\section{Authors' contributions}

MI, IC, NK, PC, RR designed the study. MI, CCS, XS, JM, RS, XC, AV performed the experiments. MI, CCS, JCS, XC, MMV, NK, PC performed the statistics. MI, CCS, JCS, DL, IC, XC, MMV, NK, PC, RR interpreted the results. ALL authors read and approved the final manuscript.

\section{Funding}

This study was financially supported by International Association for the Study of Lung Cancer (IASLC) fellowship award. This study was partially supported by the Major National Science and Technology Program of China for Innovative Drug (2017ZX09101002-002-006), Key R\&D Program of Jiangsu Province (BE2018755), the Priority Academic Program Development of Jiangsu Higher Education Institutions (Integration of Chinese and Western Medicine) grant and Asociación Española Contra el Cáncer (AECC) PROYE18012ROSE

\section{Availability of data and materials}

Data is shown properly in main manuscript and supplemental files. No additional data is provided through the special link.

\section{Ethics approval and consent to participate}

This study has been approved according World Medical Association Declaration of Helsinki and institutional ethical guidelines for the research involving animal and human subjects.

\section{Consent for publication}

Not applicable.

\section{Competing interests}

The authors declare that they have no competing interests.

\section{Author details}

${ }^{1}$ Pangaea Oncology, Laboratory of Molecular Biology, Quiron-Dexeus University Institute, Barcelona, Spain. ${ }^{2}$ Laboratory of Cellular and Molecular Biology, Institute for Health Science Research Germans Trias i Pujol (IGTP), Badalona, Spain. ${ }^{3}$ Department of Surgical Oncology, Research Institute for Radiation Biology and Medicine, Hiroshima University, Hiroshima, Japan. ${ }^{4}$ IDIBELL/CReST/Translational Research Laboratori L'Hospitalet de Llobregat, Barcelona, Spain. ${ }^{5}$ Hospital of Integrated Traditional Chinese and Western Medicine, Nanjing University of Chinese Medicine, Nanjing, China. ${ }^{6}$ Laboratory of Cellular and Molecular Biology, Jiangsu Province Academy of Traditional Chinese Medicine, Nanjing, China. ${ }^{7}$ Collaborative Innovation Center for Cancer Medicine, Nanjing Medical University, Nanjing, China. ${ }^{8}$ Istituto Scientifico Romagnolo per lo Studio e la Cura dei Tumori (IRST) IRCCS, Meldola, Italy. ${ }^{9}$ Institute of Oncology Rosell (IOR), Quiron-Dexeus University Institute, Barcelona, Spain. ${ }^{10}$ Institut d'Investigació en Ciències de la Salut Germans Trias i Pujol Campus Can Ruti (Edifici Muntanya), Ctra. de Can Ruti, Cami de les Escoles s/n, Badalona, 08916 Barcelona, Spain.

Received: 31 July 2019 Accepted: 24 September 2019

Published online: 28 October 2019

\section{References}

1. Govindan R, Ding $L$, Griffith $M$, et al. Genomic landscape of non-small cell lung cancer in smokers and never-smokers. Cell. 2012;150(6):1121-34.

2. Rosell R, Bivona TG, Karachaliou N. Genetics and biomarkers in personalisation of lung cancer treatment. Lancet. 2013;382(9893):720-31.

3. Paz-Ares L, Luft A, Vicente $D$, et al. Pembrolizumab plus chemotherapy for squamous non-small-cell lung Cancer. N Engl J Med. 2018;379(21):2040-51.

4. Gandhi L, Rodriquez-Abreu D, Gadgeel S, et al. Pembrolizumab plus chemotherapy in metastatic non-small-cell lung Cancer. N Engl J Med. 2018, 378(22):2078-92.

5. Mok TSK, Wu YL, Kudaba I, et al. Pembrolizumab versus chemotherapy for previously untreated, PD-L1-expressing, locally advanced or metastatic nonsmall-cell lung cancer (KEYNOTE-042): a randomised, open-label, controlled, phase 3 trial. Lancet. 2019;393(10183):1819-30.

6. Reck M, Mok TSK, Nishio M, et al. Atezolizumab plus bevacizumab and chemotherapy in non-small-cell lung cancer (IMpower150): key subgroup 
analyses of patients with EGFR mutations or baseline liver metastases in a randomised, open-label phase 3 trial. Lancet Respir Med. 2019;7(5):387-401.

7. Lee CK, Man J, Lord S, et al. Checkpoint inhibitors in metastatic EGFRmutated non-small cell lung Cancer-a meta-analysis. J Thorac Oncol. 2017; 12(2):403-7.

8. Regala RP, Weems C, Jamieson L, et al. Atypical protein kinase C iota is an oncogene in human non-small cell lung cancer. Cancer Res. 2005;65(19): 8905-11.

9. Justilien V, Fields AP. Ect2 links the PKCiota-Par6alpha complex to Rac1 activation and cellular transformation. Oncogene. 2009;28(41):3597-607.

10. Halaoui R, McCaffrey L. Rewiring cell polarity signaling in cancer. Oncogene. 2015;34(8):939-50.

11. Luo Q, Tang $L$, Lin H, et al. The oncogenic role of PKCiota gene amplification and overexpression in Chinese non-small cell lung cancer. Lung Cancer. 2014;84(2):190-5.

12. Regala RP, Thompson EA, Fields AP. Atypical protein kinase C iota expression and aurothiomalate sensitivity in human lung cancer cells. Cancer Res. 2008;68(14):5888-95.

13. Li H, Hu J, Wu S, et al. Auranofin-mediated inhibition of PI3K/AKT/mTOR axis and anticancer activity in non-small cell lung cancer cells. Oncotarget. 2016; 7(3):3548-58.

14. $\mathrm{Hu}$ J, Zhang $\mathrm{H}$, Cao M, et al. Auranofin enhances Ibrutinib's anticancer activity in EGFR-mutant lung adenocarcinoma. Mol Cancer Ther. 2018;17(10):2156-63.

15. Ali SA, Justilien $V$, Jamieson $L$, et al. Protein kinase Ciota drives a NOTCH3dependent stem-like phenotype in mutant KRAS lung adenocarcinoma. Cancer Cell. 2016;29(3):367-78.

16. Justilien $V$, Ali SA, Jamieson $L$, et al. Ect2-dependent rRNA synthesis is required for KRAS-TRP53-driven lung adenocarcinoma. Cancer Cell. 2017; 31(2):256-69.

17. Yang Z, Wang $H$, Xia L, et al. Overexpression of PAK1 correlates with aberrant expression of EMT markers and poor prognosis in non-small cell lung Cancer. J Cancer. 2017;8(8):1484-91.

18. Chen MJ, Wu DW, Wang YC, et al. PAK1 confers chemoresistance and poor outcome in non-small cell lung cancer via beta-catenin-mediated stemness. Sci Rep. 2016;6:34933.

19. Lu H, Liu S, Zhang G, et al. PAK signalling drives acquired drug resistance to MAPK inhibitors in BRAF-mutant melanomas. Nature. 2017;550(7674):133-6.

20. Wu DW, Wu TC, Chen CY, et al. PAK1 is a novel therapeutic target in tyrosine kinase inhibitor-resistant lung adenocarcinoma activated by the PI3K/AKT signaling regardless of EGFR mutation. Clin Cancer Res. 2016; 22(21):5370-82.

21. Jacobsen K, Bertran-Alamillo J, Molina MA, et al. Convergent Akt activation drives acquired EGFR inhibitor resistance in lung cancer. Nat Commun. 2017:8(1):410.

22. Ichihara $E$, Westover $D$, Meador $C B$, et al. SFK/FAK signaling attenuates Osimertinib efficacy in both drug-sensitive and drug-resistant models of EGFR-mutant lung Cancer. Cancer Res. 2017;77(11):2990-3000.

23. Karachaliou N, Chaib I, Cardona AF, et al. Common co-activation of AXL and CDCP1 in EGFR-mutation-positive non-smallcell lung Cancer associated with poor prognosis. EBioMedicine. 2018;29:112-27.

24. Lei $M$, Lu W, Meng $W$, et al. Structure of PAK1 in an autoinhibited conformation reveals a multistage activation switch. Cell. 2000;102(3): 387-97.

25. Jha RK, Strauss CE. 3D structure analysis of PAKs: a clue to the rational design for affinity reagents and blockers. Cell Logist. 2012;2(2):69-77.

26. Wong LL, Lam IP, Wong TY, et al. IPA-3 inhibits the growth of liver cancer cells by suppressing PAK1 and NF-kappaB activation. PLoS One. 2013;8(7):e68843.

27. Chou TC. Drug combination studies and their synergy quantification using the Chou-Talalay method. Cancer Res. 2010;70(2):440-6.

28. Parrini MC, Camonis J, Matsuda M, et al. Dissecting activation of the PAK1 kinase at protrusions in living cells. J Biol Chem. 2009;284(36):24133-43.

29. Lazzari C, Verlicchi A, Gkountakos A, et al. Molecular bases for combinatorial treatment strategies in patients with KRAS mutant lung adenocarcinoma and squamous cell lung carcinoma. Pulmonary Therapy. 2016;2(1):1-18.

30. Gong K, Guo G, Gerber DE, et al. TNF-driven adaptive response mediates resistance to EGFR inhibition in lung cancer. J Clin Invest. 2018;128(6):2500-18.

31. Prudnikova TY, Villamar-Cruz O, Rawat SJ, et al. Effects of p21-activated kinase 1 inhibition on 11q13-amplified ovarian cancer cells. Oncogene. 2016;35(17):2178-85.
32. Maruta H, Ahn MR. From bench (laboratory) to bed (hospital/home): how to explore effective natural and synthetic PAK1-blockers/longevity-promoters for cancer therapy. Eur J Med Chem. 2017;142:229-43.

33. Ong CC, Jubb AM, Haverty PM, et al. Targeting p21-activated kinase 1 (PAK1) to induce apoptosis of tumor cells. Proc Natl Acad Sci U S A. 2011; 108(17):7177-82

34. Jagadeeshan S, Sagayaraj RV, Paneerselvan N, et al. Toxicity and antiangiogenicity evaluation of Pak1 inhibitor IPA-3 using zebrafish embryo model. Cell Biol Toxicol. 2017;33(1):41-56.

35. Wang $Y$, Wang $S$, Lei $M$, et al. The p21-activated kinase 1 (Pak1) signalling pathway in cardiac disease: from mechanistic study to therapeutic exploration. Br J Pharmacol. 2018;175(8):1362-74

36. DeSantiago J, Bare DJ, Varma D, et al. Loss of p21-activated kinase 1 (Pak1) promotes atrial arrhythmic activity. Heart Rhythm. 2018;15(8):1233-41.

37. Capparelli EV, Bricker-Ford R, Rogers MJ, et al. Phase I Clinical Trial Results of Auranofin, a Novel Antiparasitic Agent. Antimicrob Agents Chemother. 2016;61(1).

38. Bernstein ILBD, Dubb JW, Faiferman I, Wallin B. A placebo-controlled multicenter study of auranofin in the treatment of patients with corticosteroid-dependent asthma. J Allergy Clin Immunol. 1996;98(2):317-24.

39. Mansfield AS, Fields AP, Jatoi A, et al. Phase I dose escalation study of the PKCiota inhibitor aurothiomalate for advanced non-small-cell lung cancer, ovarian cancer, and pancreatic cancer. Anti-Cancer Drugs. 2013; 24(10):1079-83.

40. Zhang Y, Zhou X, Li Y, et al. Inhibition of maternal embryonic leucine zipper kinase with OTSSP167 displays potent anti-leukemic effects in chronic lymphocytic leukemia. Oncogene. 2018;37(41):5520-33.

41. Chung S, Suzuki H, Miyamoto T, et al. Development of an orallyadministrative MELK-targeting inhibitor that suppresses the growth of various types of human cancer. Oncotarget. 2012;3(12):1629-40.

42. Muller J, Bolomsky A, Dubois S, et al. Maternal embryonic leucine zipper kinase inhibitor OTSSP167 has preclinical activity in multiple myeloma bone disease. Haematologica. 2018;103(8):1359-68.

43. Kong $\mathrm{JH}$, Khoury HJ, Kim AS, et al. The safety of Bosutinib for the treatment of chronic myeloid leukemia. Expert Opin Drug Saf. 2017;16(10):1203-9.

44. Zhu X, Gao JJ, Landao-Bassonga E, et al. Thonzonium bromide inhibits RANKL-induced osteoclast formation and bone resorption in vitro and prevents LPS-induced bone loss in vivo. Biochem Pharmacol. 2016;104: 118-30.

45. Yap TA, Walton MI, Grimshaw KM, et al. AT13148 is a novel, oral multi-AGC kinase inhibitor with potent pharmacodynamic and antitumor activity. Clin Cancer Res. 2012;18(14):3912-23.

46. Lu YC, Lee YR, Liao JD, et al. Reversine induced multinucleated cells, cell apoptosis and autophagy in human non-small cell lung Cancer cells. PLoS One. 2016;11(7):e0158587.

47. Zhou GL, Zhuo Y, King CC, et al. Akt phosphorylation of serine 21 on Pak1 modulates Nck binding and cell migration. Mol Cell Biol. 2003; 23(22):8058-69.

48. Isakov N. Protein kinase C (PKC) isoforms in cancer, tumor promotion and tumor suppression. Semin Cancer Biol. 2018:48:36-52.

49. Zhang X, Selvaraju K, Saei AA, et al. Repurposing of auranofin: Thioredoxin reductase remains a primary target of the drug. Biochimie. 2019;162:46-54

\section{Publisher's Note}

Springer Nature remains neutral with regard to jurisdictional claims in published maps and institutional affiliations.

Ready to submit your research? Choose BMC and benefit from:

- fast, convenient online submission

- thorough peer review by experienced researchers in your field

- rapid publication on acceptance

- support for research data, including large and complex data types

- gold Open Access which fosters wider collaboration and increased citations

- maximum visibility for your research: over $100 \mathrm{M}$ website views per year

At BMC, research is always in progress.

Learn more biomedcentral.com/submissions 